\title{
Sintilimab With Chemotherapy as First-line Treatment for Locally Advanced or Metastatic Squamous Non-small-cell Lung Cancer: Real-world Data Study
}

\section{Xinqing Lin}

First Affiliated Hospital of Guangzhou Medical University

\section{Haiyi Deng}

First Affiliated Hospital of Guangzhou Medical University

\section{Suyang Li}

First Affiliated Hospital of Guangzhou Medical University

\section{Xiaohong Xie}

First Affiliated Hospital of Guangzhou Medical University

\section{Chao Chen}

First Affiliated Hospital of Guangzhou Medical University

\section{Longqiu Cai}

First Affiliated Hospital of Guangzhou Medical University

\section{Yilin Yang}

First Affiliated Hospital of Guangzhou Medical University

\section{Guihuan Qiu}

First Affiliated Hospital of Guangzhou Medical University

\section{Zhanhong Xie}

First Affiliated Hospital of Guangzhou Medical University

\section{Yinyin Qin}

First Affiliated Hospital of Guangzhou Medical University

\section{Ming Liu}

The First Affiliated Hospital of Guangzhou Medical Univercity

Chengzhi Zhou ( $\nabla$ doctorzcz@163.com)

First Affiliated Hospital of Guangzhou Medical University https://orcid.org/0000-0003-0029-6879

\section{Research Article}

Keywords: Squamous non-small-cell lung cancer, Sintilimab, Chemotherapy, Real-world

Posted Date: October 25th, 2021 
DOI: https://doi.org/10.21203/rs.3.rs-991261/v1

License: (c) (1) This work is licensed under a Creative Commons Attribution 4.0 International License. Read Full License

Version of Record: A version of this preprint was published at Journal of Cancer Research and Clinical Oncology on February 10th, 2022. See the published version at https://doi.org/10.1007/s00432-02103903-0. 


\section{Abstract}

\section{Purpose:}

The ORIENT-12 study demonstrated the promising results of sintilimab combined with gemcitabine and platinum (GP) therapy in squamous non-small-cell lung cancer (sqNSCLC) patients. However, the efficacy of sintilimab plus paclitaxel/nab-paclitaxel and platinum (TP) in sqNSCLC is not yet known.

\section{Methods:}

Real-life data were collected from patients with untreated locally advanced or metastatic sqNSCLC who were treated with sintilimab plus TP (arm A) or sintilimab plus GP (arm B) between January 2019 and January 2021.

\section{Results:}

A total of 52 patients were included ( $\operatorname{arm~} A, n=32$ and arm $B, n=20)$. After a median follow-up of 12.1 months, the median PFS was 10.9 months ( $95 \%$ confidence interval [Cl], 5.0 to 16.7) in arm A and 7.5 months $(95 \% \mathrm{Cl}, 4.0-10.9)$ in arm $\mathrm{B}$ (hazard ratio $[\mathrm{HR}], 0.64 ; 95 \% \mathrm{Cl}, 0.30$ to $1.4 ; \mathrm{P}=0.24)$. The median overall survival was 20.1 months $(95 \% \mathrm{Cl}, 13.6$ to 26.6$)$ in arm $\mathrm{A}$ and 16.3 months ( $95 \% \mathrm{Cl}, 2.9$ to 29.6$)$ in $\operatorname{arm~B}(\mathrm{HR}, 0.69 ; 95 \% \mathrm{Cl}, 0.26-1.84 ; \mathrm{P}=0.46)$. The overall response rate was $59.4 \%$ in arm $\mathrm{A}$ and $40.0 \%$ in arm B. Adverse events of grade 3 or higher occurred in $37.5 \%$ of the patients in arm $A$ and $55.0 \%$ of the patients in arm B.

\section{Conclusions:}

Sintilimab-TP exhibits similar clinical benefits compared with sintilimab-GP in patients with untreated advanced or metastatic sqNSCLC.

\section{Introduction}

Primary lung cancer is the second most commonly diagnosed cancer with the highest mortality, worldwide (Sung et al. 2021). Squamous non-small cell lung cancer (sqNSCLC) accounts for $20-30 \%$ of all lung cancers (Socinski et al. 2016). Anti-angiogenesis therapy and targeted therapy can significantly prolong the survival time of non-squamous NSCLC, while they demonstrate moderate benefits for sqNSCLC (Wang and Li 2016). Immune checkpoint inhibitors (ICls) including programmed cell death protein 1/programmed cell death ligand 1 (PD-1/PD-L1) inhibitors significantly improve the outcomes of patients with sqNSCLC (Chen et al. 2019). The phase 3 CheckMate 017 study (Brahmer et al. 2015) demonstrated that nivolumab monotherapy, which was used as a second-line therapy compared with docetaxel treatment, remarkably improved treatment efficacy in patients with advanced sqNSCLC. Subsequently, the KEYNOTE-024 (Reck et al. 2016) and KEYNOTE-042 (Mok et al. 2019) studies confirmed the clinical benefit of ICI monotherapy in the first-line treatment of NSCLC regardless of the histological sub-groups. However, the objective response rate (ORR) of ICI monotherapy was 
unsatisfactory and was related to the expression level of PD-L1. Novel therapies that improve response and long-term efficacy are urgently required. The antitumor efficacy of ICls with paclitaxel/nab-paclitaxel and platinum as the first-line treatment of sqNSCLC has been confirmed by a series of studies, such as KEYNOTE 407 (Paz-Ares et al. 2018), IMpower131 (Jotte et al. 2020) and RATIONALE 307 (Wang et al. 2021).

Sintilimab is a highly selective, fully human monoclonal antibody that blocks the interactions of PD-1 and its ligands and possesses a strong anti-tumor response (Zhang et al. 2020). The ORIENT-12 (NCT03629925) was a randomized, double-blind, phase III study, which indicated that sintilimab combined with gemcitabine and platinum (GP) treatment significantly prolonged progression-free survival (PFS) compared with that of GP as the first-line treatment of locally advanced or metastatic sqNSCLC (median PFS: 5.5 months vs. 4.9 months; hazard ratios [HR], 0.536; 95\% confidence intervals [Cl], 0.422-0.681; $p<0.00001$ ) (Zhou et al. 2021). Paclitaxel/nanoparticle albumin-bound [nab]paclitaxel plus platinum (TP) are the standard regimens for first-line treatment of sqNSCLC. However, the efficacy of sintilimab combined with paclitaxel/nab-paclitaxel and platinum in sqNSCLC is not yet known.

The present study aimed to investigate the efficacy and safety of sintilimab with chemotherapy (paclitaxel/nab-paclitaxel+platinum or gemcitabine+platinum) in real-world patients with locally advanced or metastatic sqNSCLC.

\section{Materials And Methods}

\section{Patients}

The present study was a retrospective study conducted at the First Affiliated Hospital of Guangzhou Medical University. The medical records of patients who received their initiated therapy between January 2019 and January 2021 were reviewed. The patients were eligible for enrollment if they had pathologically confirmed sqNSCLC without sensitizing epidermal growth factor receptor (EGFR) or anaplastic lymphoma kinase (ALK) mutations, incurable stage IIIB-IV tumors (according to the 8th edition AJCC/IASLC classification (Goldstraw et al. 2016)) or at least one measurable lesion according to the Response Evaluation Criteria in Solid Tumors (RECIST), version 1.1 (Eisenhauer et al. 2009). Patients who were treated with sintilimab plus paclitaxel/nab-paclitaxel and platinum (sintilimab-TP, arm A) or sintilimab plus gemcitabine and platinum (sintilimab-GP, arm B) in the first line were recruited. Patients younger than 18 years old, who had received previous therapies for locally advanced or metastatic disease, or had the active autoimmune disease were excluded.

\section{Data collection and outcome assessment}

The patient data were collected retrospectively from medical files and included patient demographics, the ECOG PS, PD-L1 expression levels, tumor response to ICls, and adverse events (AEs). 
The ECOG PS was evaluated prior to treatment initiation. The evaluations of the clinical responses were performed by investigators according to the RECIST version 1.1. ORR was defined as the proportion of patients achieving complete response (CR) and partial response (PR). The disease control rate (DCR) corresponds to all cases with CR, PR and stable disease (SD). The time to response (TTR) was defined as the time from the initiation of sintilimab to the first documented CR or PR. PFS was estimated as the duration from the initiation of sintilimab to disease progression or patient death. The overall survival (OS) was defined as the time from the initiation of sintilimab to patient death. AEs were graded according to the National Cancer Institute Common Terminology Criteria for Adverse Events (version 4.0). PFS was the primary endpoint. OS, ORR, DCR and TTR were the secondary endpoints. The final follow-up time was April 31, 2021.

\section{Statistical analysis}

The clinical and tumor characteristics were summarized as medians and ranges for continuous variables and as frequencies and percentages for categorical variables. An independent-samples t-test or the Mann-Whitney $U$ test was used to analyze continuous variables. The differences in the continuous variables were assessed using either the Chi-square $\left(\chi^{2}\right)$ or Fisher's exact test. The Kaplan-Meier survival estimates were calculated for PFS and OS with 95\% Cl and the log-rank test was used to evaluate between-group differences. The follow-up time was estimated using the reverse Kaplan-Meier method. Cox regression was applied to calculate the $\mathrm{HR}$ with a $95 \% \mathrm{Cl}$ of factors associated with PFS. All P values were based on the two-sided hypothesis test and a P-value $<0.05$ was considered to indicate statistically significant differences. All analyses were conducted using IBM SPSS Statistics (Armonk, NY), version 25.

\section{Results}

\section{Patients}

In total, 52 patients were eligible and enrolled in the present study. The median age of all patients was 61 (range, 46-84) years. The majority of the patients were men (88.5\%) and exhibited ECOG performance status of $1(75.0 \%)$ (Table 1$)$.

Arm A (sintilimab-TP) included 32 (61.5\%) patients and arm B (sintilimab-GP) 20 (38.5\%). In arm A, 20 patients received nab-paclitaxel and 12 received paclitaxel. Baseline demographic and disease characteristics exhibited no significant differences between the groups (Table 1). The overall median follow-up for this analysis was 12.1 months. A median of 4 doses (range, 1-20) of arm A and 6 doses (range, 3-34) of arm B was used. As of April 31 2021, a total of 17 patients (53.1\%) in the sintilimab-TP group and 7 patients (35.0\%) in the sintilimab-GP group were continuing treatment.

\section{Efficacy}

The confirmed ORR was 59.4\% (95\% Cl, 41.4-77.4) in arm A and 40.0\% (95\% Cl, 16.5-63.5) in arm B (P = 0.24 ; Table 2 and figure 1). In addition, $6.4 \%$ of the patients who received sintilimab-TP and $5.0 \%$ of those 
who received sintilimab-GP exhibited disease progression (Table 2 and Fig.1). The DCR was $93.6 \%$ in arm A and $95.0 \%$ in arm B. The median TTR was 2.0 months (range: 0.8-6.7) with arm A and 1.9 months (range: $0.7-3.0$ ), respectively (Table 2 ).

A total of 28 events of progression or death were noted and the median PFS was estimated to 10.9 months $(95 \% \mathrm{Cl}, 5.0$ to 16.7$)$ in arm A and 7.5 months ( $95 \% \mathrm{Cl}, 4.0-10.9)$ in arm B (HR for PFS, $0.64 ; 95 \%$ $\mathrm{Cl}, 0.30-1.4 ; \mathrm{P}=0.24 ;$ Fig.2a). The 12-month PFS rate was $44.6 \%$ with TP and $27.1 \%$ with GP. Across subgroups that were analyzed, the two treatment groups exhibited similar PFS benefits (figure 2B). However, male patients tended to benefit from arm A over arm B (median PFS, 13.9 months [95\% Cl, 7.420.5] vs. 7.5 months [3.7-11.2]; $\mathrm{HR}, 0.51$ [95\% Cl, 0.23-1.12]; $\mathrm{P}=0.093$; Fig.2b).

The median OS was 20.1 months $(95 \% \mathrm{Cl}, 13.6-26.6)$ in arm $\mathrm{A}$ and 16.3 months $(95 \% \mathrm{Cl}, 2.9-29.6)$ in arm $B(H R, 0.69 ; 95 \% \mathrm{Cl}, 0.26-1.84 ; \mathrm{P}=0.46)$ (Fig.3a), with a total of 19 events in the treatment population. The OS rate at 1 year was $72.9 \%$ in arm A and $53.3 \%$ in arm B. Similar OS benefits were observed in all prespecified subgroups of the two treatment groups (Fig.3b).

\section{Safety}

AEs of any cause occurred in all patients in the two treatment groups (Table 3). AEs of grade 3 or higher occurred in $37.5 \%$ of the patients in arm $A$ and $55.0 \%$ of the patients in the arm B. The AEs led to the discontinuation of all treatment components in $3.1 \%$ and $10.0 \%$ of arms $A$ and $B$, respectively and to the discontinuation of sintilimab in $12.5 \%$ and $10.0 \%$ of arms $A$ and $B$, respectively. No toxicity-related deaths occurred. The most common AEs in arm A were anemia (68.8\%), decreased white blood cell count (37.5\%), increased alanine aminotransferase $(31.3 \%)$ and decreased neutrophil count $(25.0 \%)$, whereas the most common AEs in arm B were anemia (75.0\%), decreased white blood cell count $(40.0 \%)$, decreased neutrophil count (30.0\%) and decreased platelet count $(20.0 \%)$.

Immune-mediated adverse events (irAEs) occurred in 12 of 32 patients (40.6\%) in arm A and in 9 of 20 patients $(45.0 \%)$ in arm B; grade 3 or higher AEs occurred in 3 patients $(9.1 \%)$ and one patient $(5.0 \%)$, respectively. A higher number of hematological AEs was noted in arm B than in arm A, while the number of cases with hyperthyroidism, myositis, peripheral neuropathy and immune-mediated pneumonitis was higher in arm A than that in arm B.

\section{Discussion}

This real-world retrospective observational study demonstrated that sintilimab combined with TP had similar clinical benefit compared with sintilimab plus GP in patients with untreated advanced or metastatic sqNSCLC.

In the phase 3 ORIENT-12, sintilimab in combination with GP revealed a significantly prolonged PFS compared with chemotherapy alone in patients with sqNSCLC (Zhou et al. 2021). The results of phase 3 trials suggested that the combination of PD-1 inhibitors and TP had greater efficacy than that of TP 
monotherapy (Jotte et al. 2020; Paz-Ares et al. 2018; Wang et al. 2021). Therefore, sintilimab with TP may have similar or even improved efficacy than sintilimab combined with GP.

In the present study, the sintilimab-TP group exhibited higher ORR (59.4\% vs. $40.0 \%), 12$-month OS rate (72.9\% vs. 53.3\%), PFS (median, 10.9 vs. 7.5 months; HR = 0.64) and OS (median, 20.1 vs. 16.3 months; $H R=0.69)$ than the sintilimab-GP group. However, the results were not significantly different. The PFS and ORR of arm B were consistent with those of sintilimab-GP in the ORIENT-12 study (median PFS, 5.5 months; ORR, 44.7\%) (Zhou et al. 2021). In addition, the outcomes of arm A observed in the present study were comparable to those reported in the KEYNOTE-407 (median PFS, 6.4 months; median 0S, 17.1 months) (Paz-Ares et al. 2018), IMpower131 (median PFS, 6.3 months; median OS, 14.2 months) (Jotte et al. 2020), and RATIONALE 307 (median PFS, 7.6 months) (Wang et al. 2021) studies.

Sintilimab with TP or GP therapy was well tolerated, with a low proportion of grade 3 or higher AEs and discontinuation of treatment due to AEs. In addition, the rate of discontinuation of treatment due to AEs was lower in the sintilimab-TP group than that in the sintilimab-GP group (3.1\% vs. $10.0 \%)$. The high frequencies of $A E s$ in both groups included anemia, decreased white blood cell count and decreased neutrophil count. The incidence of these hematological AEs was considered to be related to chemotherapy and a lower number of patients were treated with sintilimab-TP than with sintilimab-GP. However, non-hematological AEs were higher in arm A than arm B. A previous study reported higher number of cases with anemia, neutropenia and thrombocytopenia in the TP group than that of the GP group. Notably the number of cases with grade 3 or 4 anemia and neutropenia was significantly higher in the TP group compared with that of the GP group (Mudad et al. 2017). The AE profile observed in the present study was consistent with our expectations and no new adverse events were observed. The majority of AEs were resolved or improved and were manageable.

The present study exhibits certain limitations. Firstly, it was a retrospective observational study with a small sample size. A large-scale prospective cohort study should be conducted to further explore these findings. Secondly, the results provided only a narrow time window with limited follow-up for certain patients. Our follow-up results will provide a more comprehensive analysis.

In conclusion, the present study revealed that the effectiveness of sintilimab plus platinum and gemcitabine or paclitaxel/nab-paclitaxel in a real-world setting was comparable to that reported in clinical trials. The treatment groups exhibited similar efficacy with regard to patients with untreated advanced or metastatic sqNSCLC, with safety profiles. Sintilimab combined with paclitaxel/nab-paclitaxel and platinum may be a novel option for the treatment of sqNSCLC patients.

\section{Abbreviations}

GP, gemcitabine+platinum; sqNSCLC, squamous non-small-cell lung cancer;TP, paclitaxel/nab-paclitaxel and platinum; PFS, progression-free survival; $\mathrm{HR}$, hazard ratio; $\mathrm{Cl}$, confidence interval; $\mathrm{PD}-1$, programmed cell death protein 1; PD-L1, programmed cell death ligand 1; ORR, objective response rate; ICls, immune checkpoint inhibitors; EGFR, epidermal growth factor receptor; ALK, Anaplastic Lymphoma Kinase; 
RECIST, Response Evaluation Criteria in Solid Tumors; CR, complete response; PR, partial response; DCR, disease control rate; SD, stable disease; TTR, time to response; OS, overall survival; AEs, adverse Events; ECOG PS, Eastern Cooperative Oncology Group performance status

\section{Statements And Declarations}

\section{Ethics Statement}

The studies involving human participants were reviewed and approved by the Institutional Review Board of the First Affiliated Hospital of Guangzhou Medical University (Guangzhou, Guangdong, China).

\section{Conflict of Interests}

All authors have no conflicts of interest to disclose.

\section{Author Contributions}

$X L, H D, M L$ and $C Z$ designed the study. $H D, S L, C C, Y Y$ and $G Q$ collected the patient data. $X L, H D, S L, X X$, $L C, Z X$ and $Y Q$ analyzed the data. $X L, H D, M L$ and $C Z$ drafted and revised the manuscript. All authors read and approved the final version of the manuscript.

\section{Funding}

The present study was supported by State Key Laboratory of Respiratory Disease-The open project [SKLRD-OP-202111], Fundamental and Applied Fundamental Research Project of City-School (Institute) Joint Funding Project, Guangzhou Science and Technology Bureau[202102010345], and Zhongnanshan Medical Foundation of Guangdong Province [ZNSA-2020003].

\section{Acknowledgments}

We sincerely thank the participating patients and all medical staff members.

\section{Data Availability Statement}

All datasets presented in this study are included in the article/supplementary material. Further inquiries can be directed to the corresponding author.

\section{References}

1. Brahmer $\mathrm{J}$ et al. (2015) Nivolumab versus Docetaxel in Advanced Squamous-Cell Non-Small-Cell Lung Cancer The New England journal of medicine 373:123-135 doi:10.1056/NEJMoa1504627

2. Chen RL et al. (2019) The efficacy of PD-1/PD-L1 inhibitors in advanced squamous-cell lung cancer: a meta-analysis of 3112 patients Immunotherapy 11:1481-1490 doi:10.2217/imt-2019-0101 
3. Eisenhauer EA et al. (2009) New response evaluation criteria in solid tumours: revised RECIST guideline (version 1.1) European journal of cancer (Oxford, England : 1990) 45:228-247 doi:10.1016/j.ejca.2008.10.026

4. Goldstraw P et al. (2016) The IASLC Lung Cancer Staging Project: Proposals for Revision of the TNM Stage Groupings in the Forthcoming (Eighth) Edition of the TNM Classification for Lung Cancer Journal of thoracic oncology : official publication of the International Association for the Study of Lung Cancer 11:39-51 doi:10.1016/j.jtho.2015.09.009

5. Jotte R et al. (2020) Atezolizumab in Combination With Carboplatin and Nab-Paclitaxel in Advanced Squamous NSCLC (IMpower131): Results From a Randomized Phase III Trial Journal of thoracic oncology : official publication of the International Association for the Study of Lung Cancer 15:13511360 doi: $10.1016 /$ j.jtho.2020.03.028

6. Mok TSK et al. (2019) Pembrolizumab versus chemotherapy for previously untreated, PD-L1expressing, locally advanced or metastatic non-small-cell lung cancer (KEYNOTE-042): a randomised, open-label, controlled, phase 3 trial Lancet (London, England) 393:1819-1830 doi:10.1016/s0140-6736(18)32409-7

7. Mudad R, Patel MB, Margunato-Debay S, Garofalo D, Lal LS (2017) Comparative effectiveness and safety of nab-paclitaxel plus carboplatin vs gemcitabine plus carboplatin in first-line treatment of advanced squamous cell non-small cell lung cancer in a US community oncology setting Lung Cancer (Auckland, NZ) 8:179-190 doi:10.2147/lctt.S139647

8. Paz-Ares $L$ et al. (2018) Pembrolizumab plus Chemotherapy for Squamous Non-Small-Cell Lung Cancer The New England journal of medicine 379:2040-2051 doi:10.1056/NEJMoa1810865

9. Reck M et al. (2016) Pembrolizumab versus Chemotherapy for PD-L1-Positive Non-Small-Cell Lung Cancer The New England journal of medicine 375:1823-1833 doi:10.1056/NEJMoa1606774

10. Socinski MA et al. (2016) Clinicopathologic Features of Advanced Squamous NSCLC Journal of thoracic oncology : official publication of the International Association for the Study of Lung Cancer 11:1411-1422 doi:10.1016/j.jtho.2016.05.024

11. Sung H, Ferlay J, Siegel RL, Laversanne M, Soerjomataram I, Jemal A, Bray F (2021) Global Cancer Statistics 2020: GLOBOCAN Estimates of Incidence and Mortality Worldwide for 36 Cancers in 185 Countries CA: a cancer journal for clinicians 71:209-249 doi:10.3322/caac.21660

12. Wang $\mathrm{J}$ et al. (2021) Tislelizumab Plus Chemotherapy vs Chemotherapy Alone as First-line Treatment for Advanced Squamous Non-Small-Cell Lung Cancer: A Phase 3 Randomized Clinical Trial JAMA oncology 7:709-717 doi:10.1001/jamaoncol.2021.0366

13. Wang S, Li J (2016) Progress in Immunotherapy for Squamous Non-small Cell Lung Cancer Zhongguo fei ai za zhi = Chinese journal of lung cancer 19:682-686 doi:10.3779/j.issn.10093419.2016.10.09

14. Zhang L, Mai W, Jiang W, Geng Q (2020) Sintilimab: A Promising Anti-Tumor PD-1 Antibody Frontiers in oncology 10:594558 doi:10.3389/fonc.2020.594558 
15. Zhou C et al. (2021) Sintilimab Plus Platinum and Gemcitabine as First-Line Treatment for Advanced or Metastatic Squamous NSCLC: Results From a Randomized, Double-Blind, Phase 3 Trial (ORIENT12) Journal of thoracic oncology : official publication of the International Association for the Study of Lung Cancer 16:1501-1511 doi:10.1016/j.jtho.2021.04.011

\section{Tables}

Table 1. Characteristics of patients at baseline

\begin{tabular}{|c|c|c|c|}
\hline Characteristics & $\operatorname{Arm~A}(\%)(\mathrm{N}=32)$ & $\operatorname{Arm~B~}(\%)(N=20)$ & P-value \\
\hline \multicolumn{4}{|l|}{ Age $(y)$} \\
\hline Median (range) & $63(46-84)$ & $58(47-77)$ & 0.62 \\
\hline$\triangle 60, n(\%)$ & $11(34.4)$ & $11(55.0)$ & 0.16 \\
\hline Sex (male/ female) & $29 / 3$ & $17 / 3$ & 0.66 \\
\hline Smoking status, n (\%) & & & 0.16 \\
\hline Current/former & $16(50.0)$ & $14(70.0)$ & \\
\hline Never & $16(50.0)$ & $6(30.0)$ & \\
\hline \multicolumn{4}{|l|}{ ECOG PS, n (\%) } \\
\hline 0 & $8(25.0)$ & $5(25.0)$ & 1 \\
\hline 1 & $24(75.0)$ & $15(75.0)$ & \\
\hline Disease status, n (\%) & & & 0.38 \\
\hline$\| \mathrm{IIB} / \mathrm{IIC}$ & $21(65.6)$ & $10(50.0)$ & \\
\hline IV & $11(34.4)$ & $10(50.0)$ & \\
\hline PD-L1 TPS,n (\%) & & & 0.73 \\
\hline$\bigotimes 1 \%$ & $6(18.7)$ & $4(20.0)$ & \\
\hline $1-49 \%$ & $12(37.5)$ & $10(50.0)$ & \\
\hline$\geq 50 \%$ & 7 (21.9) & $4(20.0)$ & \\
\hline Unknown & $7(21.9)$ & $2(10.0)$ & \\
\hline \multicolumn{4}{|c|}{ Previous therapy for nonmetastatic disease } \\
\hline Neoadjuvant therapy & 0 & 1 & 0.39 \\
\hline Adjuvant therapy & $1(3.1)$ & $2(10.0)$ & 0.55 \\
\hline
\end{tabular}


ECOG PS, Eastern Cooperative Oncology Group performance status; PD-L1 TPS, programmed deathligand 1 tumor proportion score.

Table 2. Clinical activity

\begin{tabular}{|lll|}
\hline Variable & Arm A(N=32) & $\operatorname{Arm~B(N=20)}$ \\
\hline Objective response rate, $\mathrm{n}(\%)[95 \% \mathrm{Cl}]$ & $19(59.4)[41.4,77.4]$ & $8(40.0)[16.5,63.5]$ \\
\hline Best overall response, $\mathrm{n}(\%)$ & & \\
\hline Partial response & $18(58.1)$ & $8(40.0)$ \\
\hline Stable disease & $11(35.5)$ & $11(55.0)$ \\
\hline Progressive disease & $2(6.4)$ & $1(5.0)$ \\
\hline Median time to response, months (range) & $2.0(0.8,6.7)$ & $1.9(0.7,3.0)$ \\
\hline
\end{tabular}

$\mathrm{Cl}$, confidence interval.

Table 3. Adverse Events of Any Cause 


\begin{tabular}{|c|c|c|c|c|}
\hline \multirow[t]{2}{*}{ Event } & \multicolumn{2}{|c|}{$\operatorname{Arm~} \mathrm{A}(\mathrm{N}=32)$} & \multicolumn{2}{|c|}{$\operatorname{Arm} B(N=20)$} \\
\hline & $\begin{array}{l}\text { Any } \\
\text { Grade }\end{array}$ & $\begin{array}{l}\text { Grade } 3 \\
\text { or } 4\end{array}$ & $\begin{array}{l}\text { Any } \\
\text { Grade }\end{array}$ & $\begin{array}{l}\text { Grade } 3 \\
\text { or } 4\end{array}$ \\
\hline Any event & $\begin{array}{l}32 \\
(100)\end{array}$ & $12(37.5)$ & $\begin{array}{l}20 \\
(100)\end{array}$ & $11(55.0)$ \\
\hline $\begin{array}{l}\text { Event leading to discontinuation of all treatment } \\
\text { components }\end{array}$ & $1(3.1)$ & $1(3.1)$ & $2(10.0)$ & $2(10.0)$ \\
\hline Event leading to discontinuation of sintilimab & $4(12.5)$ & $3(9.4)$ & $2(10.0)$ & $2(10.0)$ \\
\hline Anemia & $\begin{array}{l}22 \\
(68.8)\end{array}$ & $1(3.1)$ & $\begin{array}{l}15 \\
(75.0)\end{array}$ & $4(20.0)$ \\
\hline White blood cell count decreased & $\begin{array}{l}12 \\
(37.5)\end{array}$ & $1(3.1)$ & $8(40.0)$ & $1(5.0)$ \\
\hline Alanine aminotransferase increased & $\begin{array}{l}10 \\
(31.3)\end{array}$ & $2(6.3)$ & $2(10.0)$ & 0 \\
\hline Neutrophil count decreased & $8(25.0)$ & $2(6.3)$ & $6(30.0)$ & $1(5.0)$ \\
\hline Platelet count decreased & $3(9.4)$ & $1(3.1)$ & $4(20.0)$ & $1(5.0)$ \\
\hline Immune-mediated pneumonitis & $3(9.4)$ & $1(3.1)$ & $1(5.0)$ & 0 \\
\hline Rash & $2(6.3)$ & 0 & $3(15.0)$ & 0 \\
\hline Hyperthyroidism & $2(6.3)$ & 0 & 0 & 0 \\
\hline Myositis & $2(6.3)$ & $1(3.1)$ & 0 & 0 \\
\hline Hypothyroidism & $1(3.1)$ & 0 & $1(5.0)$ & 0 \\
\hline Peripheral neuropathy & $1(3.1)$ & $1(3.1)$ & 0 & 0 \\
\hline Encephalitis & 0 & 0 & $1(5.0)$ & $1(5.0)$ \\
\hline
\end{tabular}

\section{Figures}




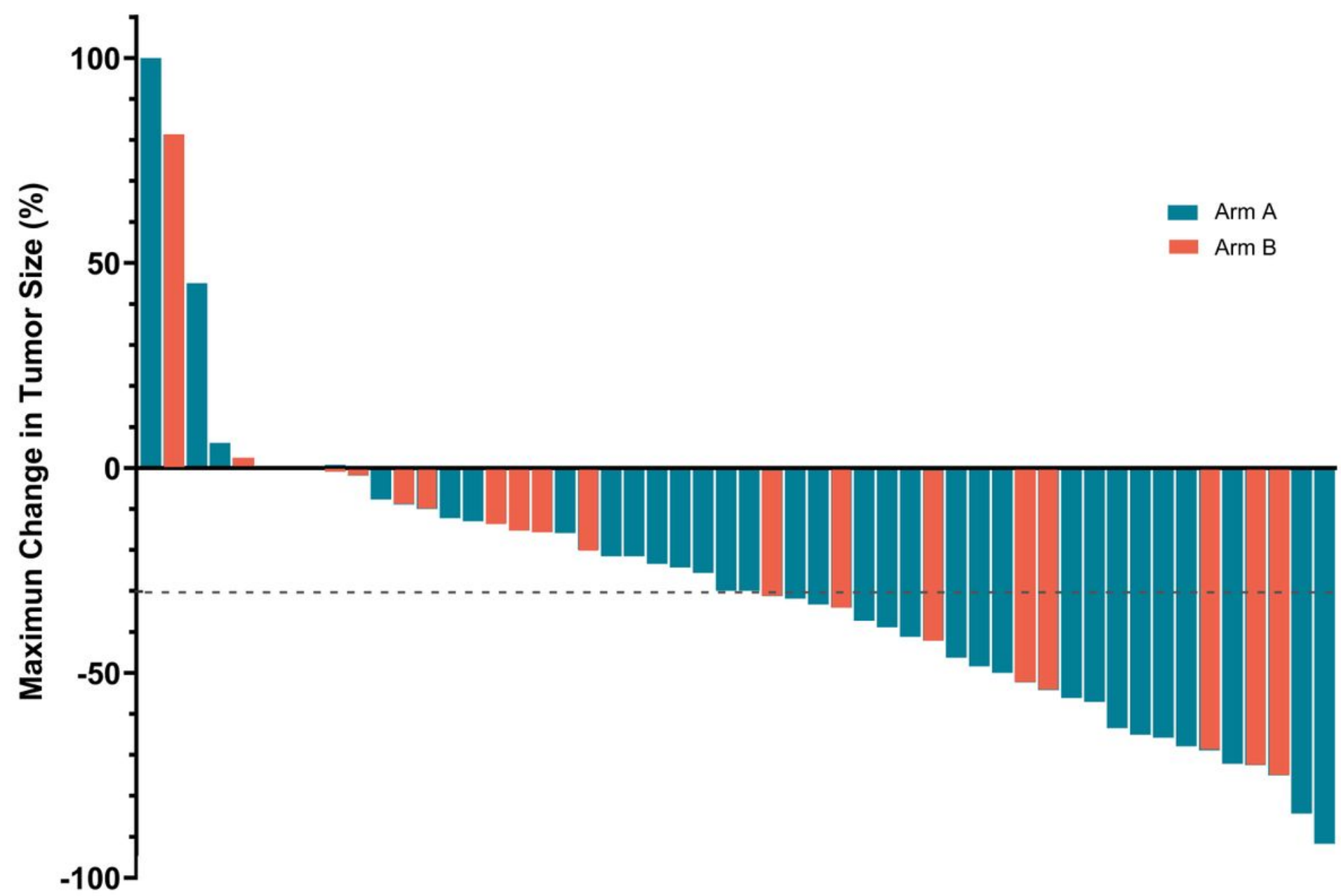

Figure 1

Maximum percentage change from baseline in target lesion measurement by Response Evaluation Criteria in Solid Tumors (RECIST) version 1.1. The positive tumor size change indicates tumor growth, whereas the negative tumor size change indicates tumor reduction. 


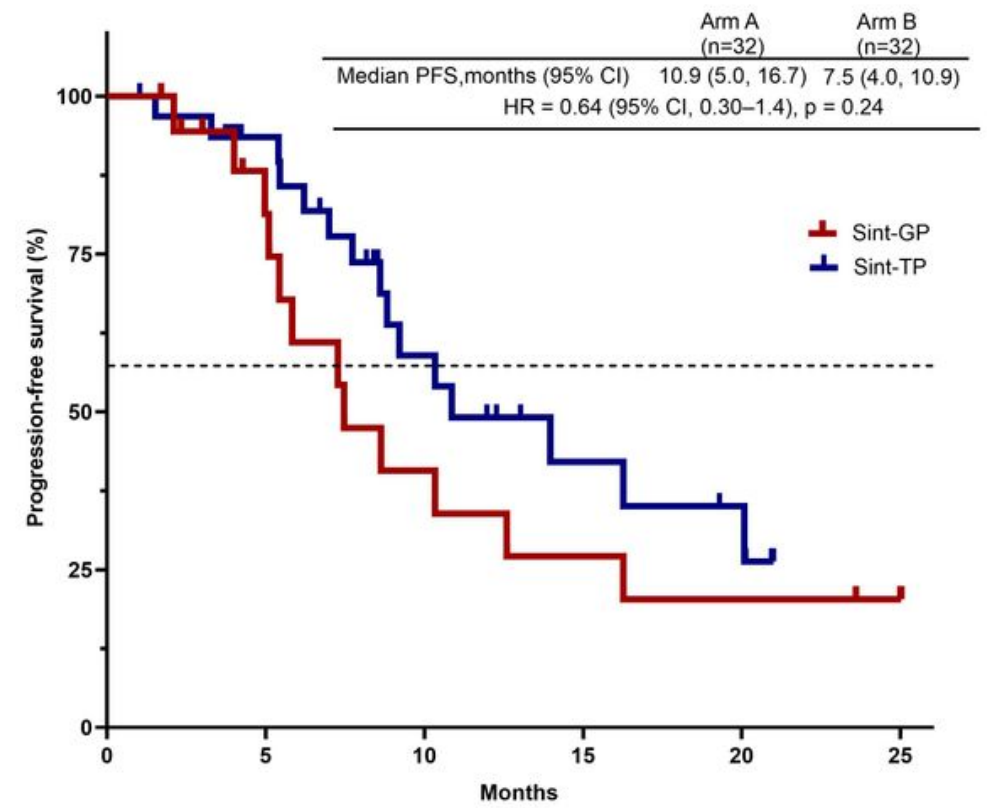

b

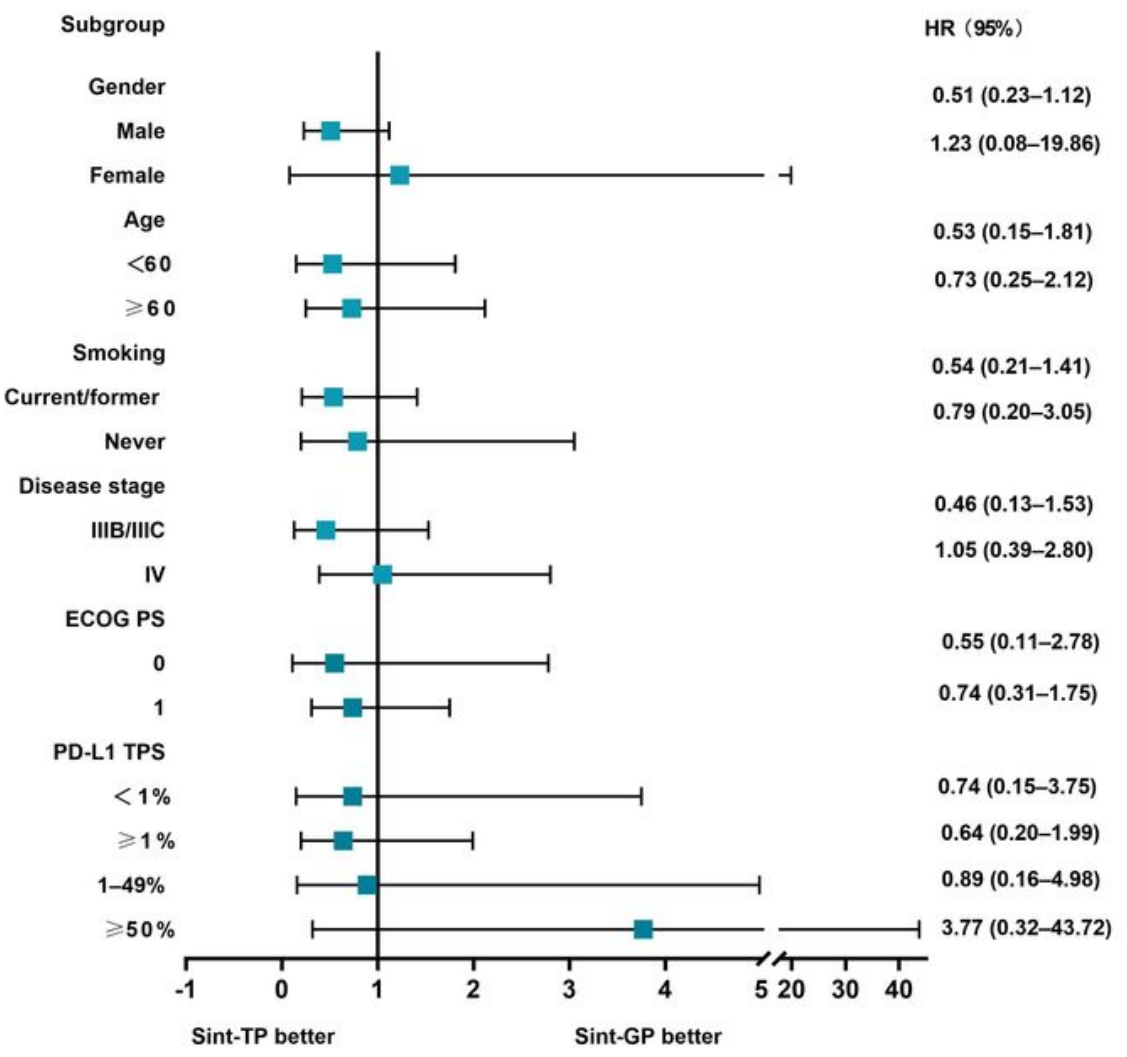

Figure 2

Kaplan-Meier curves of estimated progression-free survival (PFS) (a) and a Cox proportional hazards regression analysis of PFS in prespecified subgroups (b). $\mathrm{Cl}$, confidence interval; HR, hazard ratio; Sint, sintilimab; GP, gemcitabine and platinum; TP, paclitaxel/nab-paclitaxel and platinum; ECOG PS, Eastern Cooperative Oncology Group performance status; PD-L1 TPS, programmed death-ligand 1 tumor proportion score. 


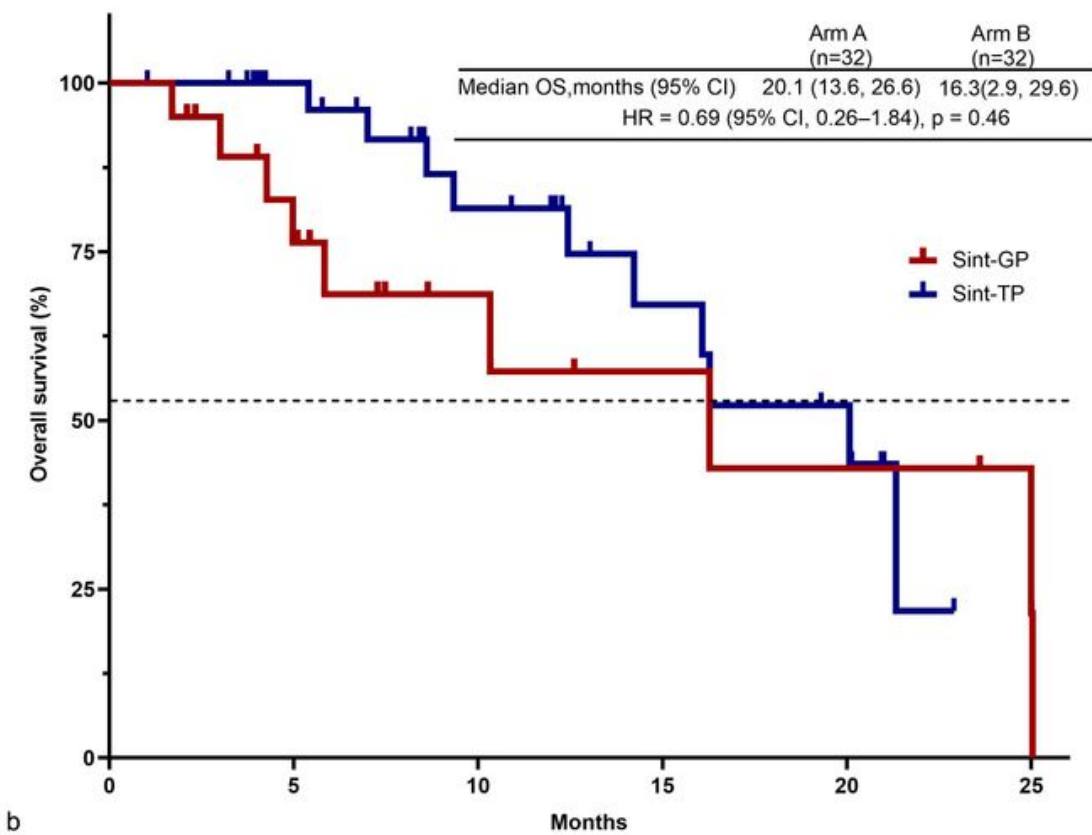

b

Subgroup

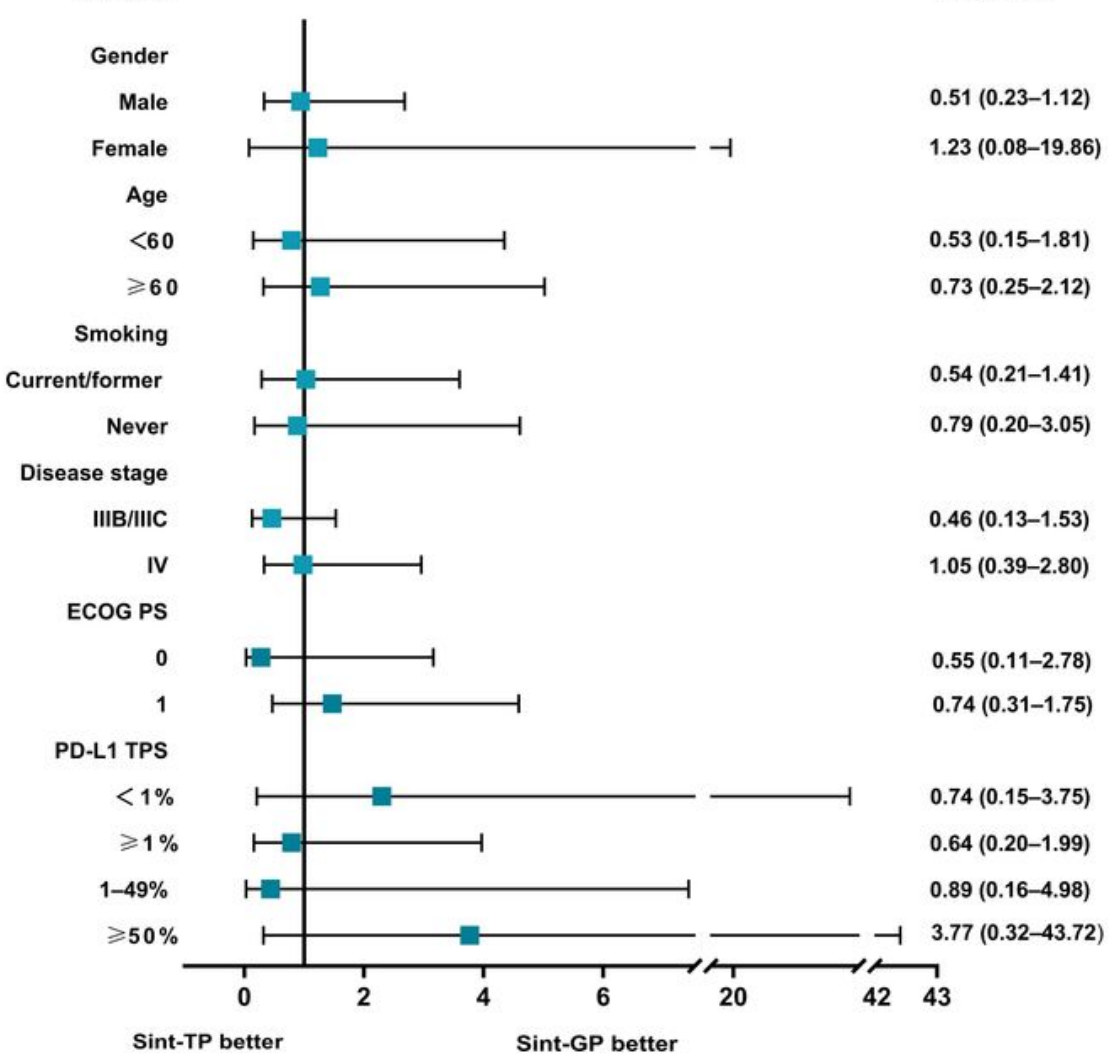

\section{Figure 3}

Kaplan-Meier survival curve analysis for overall survival (OS) (a) and analysis of OS in key prespecified subgroups (b). Cl, confidence interval; HR, hazard ratio; Sint, sintilimab; GP, gemcitabine and platinum; TP, paclitaxel/nab-paclitaxel and platinum; ECOG PS, Eastern Cooperative Oncology Group performance status; PD-L1 TPS, programmed death-ligand 1 tumor proportion score. 